\title{
The Role of Functional MRR in Understanding the Origin of Speech Delay in Auttism Spectrum Disorders
}

\author{
DOI: $10.17691 / \mathrm{stm} 2019.11,3.09$
}

Received/April 24, 2019

E.A. Kliuev, PhD Student, Department of Radiological Diagnostics;

G.E. Sheyko, MD, PhD, Assistant, Department of Medical Rehabilitation;

M.G. Dunayev, Junior Researcher, Department of Functional Diagnostics, University Clinic;

S.A. Abramov, Head of the Department of Anesthesiology and Resuscitation, Institute of Pediatrics,

University Clinic;

V.V. Dvoryaninova, Assistant, Department of Psychiatry and Medical Psychology; Junior Researcher,

Department of Functional Diagnostics, University Clinic;

O.V. Balandina, Head of the University Center of Psychology and Children Development;

N.N. Karyakin, MD, DSc, Rector;

A.N. Belova, MD, DSc, Professor, Head of the Department of Functional Diagnostics, University Clinic;

Head of the Department of Medical Rehabilitation

Privolzhsky Research Medical University, 10/1 Minin and Pozharsky Square, Nizhny Novgorod, 603005, Russia

Autism spectrum disorders (ASD) are disorders of psychic development characterized by the difficulties of social interaction and stereotyped and repetitive patterns of behavior. Rather often they are accompanied by disturbances of speech, intelligence, and adaptive behavior. Pathogenesis of ASD is still poorly studied. MRI with its latest modalities is a modern diagnostic method enabling medical providers to evaluate structural, metabolic, and functional features of brain development in this pathology.

The aim of the study was to assess the capabilities of functional MRI (fMRI) in determining pathophysiological mechanisms of delay in speech development in ASD.

Materials and Methods. A brief review of international studies is given in the article. Our own results of examining 6 preschool children with one of the ASD forms - early childhood autism and speech disorders, and 6 children of the comparison group without autism and language disturbances are also presented using $\mathrm{fMRI}$ and a block design paradigm to analyze speech perception patterns.

Results. In all children with normal speech development, bilateral symmetric spread of activation along the cortex of the entire superior temporal gyri was revealed whereas children with autism showed lateralized and limited involvement of the auditory cortex. Sevoflurane anesthesia did not influence the character of auditory zone activation.

Conclusion. The possibility of using fMRI with application of the paradigm for speech understanding to study the individual features of brain functioning in children with autism has been demonstrated. The revealed objective instrumental signs of brain activity differences in the children with autism compared to the healthy children allow the fMRI data to be considered as a potential biomarker of this disease. It has also been shown that the possibility to carry out this examination under general anesthesia makes it more acceptable and convenient for patients with childhood autism.

Key words: autism; autism spectrum disorders; ASD; magnetic resonance imaging; functional MRI; speech development.

\section{Introduction}

Autism spectrum disorders (ASD) represent a group of disintegrative disturbances of psychic developments and are characterized by a reduction of human ability to social interaction and communication as well as stereotypic behavior [1, 2]. The following nosological forms are referred to the ASD group: childhood autism (CA), atypical autism, Asperger's syndrome, and others. In recent years, a worldwide growth of ASD prevalence has been noted causing an increase of scientific works devoted to the study of etiology and pathogenesis of these disorders, nevertheless, the nature of this group of diseases remains obscure until now [1].
One of the hypotheses concerning ASD pathogenesis is the hypothesis about the role of synaptic transmission deficit determined by genetic factors and resulting in anatomical impairment of the brain cortex microstructure and in the dysfunction of its neuronal networks $[3,4]$. In the diseases of this group, disturbance of cortical interactions between various parts of the brain is observed; in this connection, the term "developmental disconnection syndrome" became applicable to ASD [5]. However, the ratio between cerebral functions, structure, and associations in different forms of autism and ASD are still poorly explored [6, 7]. Neurovisualization investigations facilitate understanding of structural and functional disorders in brain development in ASD [8].

Corresponding author: Evgeniy A. Kliuev, e-mail: eugenekluev@yandex.ru 
Magnetic resonance imaging (MRI) is one of the promising techniques of noninvasive brain study for patients with ASD [9]. Undoubtedly, functional MRI gives the greatest bulk of information relative to this disease.

Functional MRI (fMRI) provides the possibility to evaluate indirectly the degree of functional activity based on the blood flow changes in different parts of the brain [10]. Intensification of metabolism in the excited areas leads to the decrease of the concentration ratio of deoxygenated hemoglobin (paramagnetic) and oxygenated hemoglobin (diamagnetic) manifesting itself in the sharp change of magnetic properties, i.e. variations in the BOLD (blood oxygen level-dependent) signal. This parameter is assessed separately for each examined voxel and then, on the basis of similarity of time and frequency characteristics between all voxels, regions with the more active blood circulation, i.e. involved in the performance of the examined function, are programmatically isolated [10-12].

The activity of various regions is assessed during the performance of the activating task by the patient, i.e. the paradigm using the method of the task-based $\mathrm{fMRI}$ and without presenting the paradigm, i.e. at rest using resting-state fMRI [13]. The activating tasks include presentation of visual, audio, and tactile stimuli to which the examined individual responds in a definite way, actively or passively; as a result, zones involved in the performance of the tested function are mapped. When resting-state $\mathrm{fMRI}$ is registered, the paradigms are absent, the examined person must be in a maximally relaxed state enabling visualization of the basal brain activity and the connectivity of its different regions with each other [14]. Interconnection of cognitive processes and functional activity of the brain gives grounds to suggest that fMRI data could serve as informative biomarkers of ASD [6, 15].

When patients with ASD are examined by the taskbased fMRI method, it is possible to map the zones participating in the performance of a large number of functions: from pain perception [16, 17] to making socially significant decisions [18-20]. Since the disorders of the ability to communicate and specific behavior patterns are the basic characteristics of ASD, the emphasis is usually placed exactly on these functions in patients with the diseases of this group during fMRI with paradigm presentation and age-related changes of the brain should be taken into consideration $[6,15$, 21]. The superior temporal gyrus with the adjacent zones, amygdala, inferior frontal gyrus, medial prefrontal cortex [21, 22] are referred to the "social" brain zones (brain regions playing a central role in recognizing the intentions of others and in social integration); therefore, fMRI investigations in autism are mainly focused on the changes of the activity of these brain regions $[6,15]$.

Several systemic reviews have been devoted to a detailed analysis of fMRI results in autism [6, 15, 21, 23]. Practically all authors find differences in functional cerebral connections in patients with various forms of autism and healthy people, however, the results of these investigations appear often contradictory. Despite the diversity of fMRI findings in ASD, the general trend is the detection of hypoactivated "social" zones in children and adults suffering from the presence of aberrant connections between the inferior and medial frontal gyri, anterior cingulate cortex, and basal ganglia [15].

One of the most typical ASD signs is inability to keep up the verbal contact with other people resulting in communication deficits $[1,24]$. There is a great number of works devoted to clarifying neuronal mechanisms of speech development delay in the diseases of this group with the help of fMRI [25, 26]. The communicative disorders in ASD have been demonstrated to be associated with different patterns of lateralization of the speech functions with the reduction of synchronization of those brain regions which are usually involved in speech organization, and vice versa with recruiting the brain zones which do not commonly participate in the speech processes [15].

Thus, fMRI examination carried out by the American scientists [27] showed that those regions of the cortex which are considered responsible for speech and spatial orientation turned out to be less synchronized in adolescents with autism than in healthy people when they performed the tasks for identifying the meaning of the sentences written on the screen. Young people with ASD used mainly visual rather than speech strategies, and during the performance of this task hypoactivation of the left inferior frontal, left medial frontal, and left angular gyri were observed in comparison with the control group. The researchers from South Korea [28] have assessed quantitatively the degree of connection concentration in the zones of speech analyzer. In patients with ASD, this index appeared to be lower than in the control group which, in the authors' judgement, spoke of connectivity disorder in the speech zones.

Wang et al. [25] have explored the ability of children with highly functional autism to understand jokes. The attempts to understand the mentality of the utterances addressed to them in these children in contrast to the healthy ones were accompanied by hyperactivation of the right inferior frontal gyrus and both temporal areas. The authors have suggested that autistic children make greater efforts to interpret the meaning of the words which is reflected in the increased functional activity of the respective zones in the speech analyzer.

Similar results have been obtained while investigating the understanding of metaphors (hyperactivation in the thalamus, medial temporal and medial occipital gyri was observed in ASD patients [29]) and in studying the synthesis of synonyms (the examined patients showed a quicker response to the task which was seen in the more intensive activation of the additional speech zones; at the same time, a less evident left-sided lateralization was noted relative to the healthy children from the group of comparison [30]). It is supposed that during usual verbal communication those neuronal connections which are 
normally involved only when the task to be performed requires greater attention are recruited in the brain of an ASD child [26].

The authors of the systemic reviews note significant variability of findings investigating the speech analyzer in children with ASD by the fMRI method [6, 15, 21, 23]. The activity of the relevant cortical regions may be lower than in healthy people but may be higher as well which can be explained by extreme overstrain of brain resources to perform simple tasks. The zones of activation may be located in a typical way or may be lateralized in a certain hemisphere; in some cases, additional regions of the cortex are activated which is determined by a number of factors. For example, mapping of the neuronal networks within the group of individuals with ASD detects differences between genders [31].

Thus, the data found in the literature confirm great capabilities of $\mathrm{FMR}$ in detecting abnormal functioning of the brain in ASD and, at the same time, show substantial differences of these data obtained by various authors. A great diversity of the results presented may be connected with the differences in the study protocols, small examined groups, diversity of pathogenetic mechanisms of different autism forms in the concrete individuals within one common ASD group [15, 32].

In order to use fMRI data for the individual prognosis of ASD development and the disease correction, it is necessary to define different clinical ASD phenotypes and neuroimaging patterns which characterize them [7, 33]. The perspectives of autism study using fMRI are associated with multicenter continued studies using a single protocol and forming groups consisting of patients with similar clinical phenotypes and age $[6,9$, $15,21,23]$.

Separate attention should be paid to the problem of sociability of ASD patients and their inability to be motionless for a long time. A high level of anxiety and motor activity in many patients with the diseases of this group makes it necessary to use various sedation methods during the fMRI procedure: carrying out fMRI in their sleep [34, 35] or under general anesthesia [36]. Tang and Ramani [36] have analyzed in their review the results of fMRI performed by different investigators using various sedatives such as propofol, sevoflurane, isoflurane. Summing up the common experience the researchers came to the conclusion that the degree of sedation influences the reception of the BOLD signal suppressing the activity in some parts of the brain cortex. The study of Gemma et al. [37] has demonstrated that application of propofol affected speech perception. It manifested itself in the restriction of activation zones in the region of Wernicke's area and absence of activation in Broca's area. A less extended activation of the relevant cortical zones with concurrent preservation of the activity in the main neuronal networks was noted during restingstate fMRI conducted under general anesthesia [38]. Thus, anesthesia provides the possibility to perform $\mathrm{fMRI}$ on unsociable patients, however, there are some limitations: first, the use of paradigms based on the active response of the tested person is excluded; second, there is a certain modification of the activation area proper. Nevertheless, the possibility to register brain activity by the fMRI method on a child being under anesthesia signifies the necessity of further exploration of this problem in order to select optimally the concentration of the drugs and the method of their introduction for obtaining indices of brain activity as close to the wakeful state as possible.

The aim of the study was to find differences between the neuronal brain activity registered by the fMRI method with paradigm presentation for speech perception in preschool children with ASD and children without disorders of speech development.

\section{Materials and Methods}

Functional MRI was conducted on 6 children ( 5 boys and 1 girl) with CA aged from 3 years and 10 months to 6 years and 7 months; 4 children were right-handed, 1 left-handed, 1 ambidexter. All examined children had significant underdevelopment of expressive speech and experienced difficulties in understanding the speech addressed to them. On the basis of history-taking and clinical examination data, they were established the diagnosis "childhood autism" (F-84.0 according to ICD-10). The diagnosis was confirmed by the results of investigations carried out in compliance with Autism Diagnostic Observation Schedule 2, or ADOS-2, which enables medical providers to assess the degree of the disease manifestation in a child. As a result, a diagnostic category ADOS-2 ("autism", "autism spectrum", or "no autism") has been defined and the degree of autism manifestations in a child within the frames of the diagnostic category in comparison with the children having a similar level of speech development (high, moderate, low, minimal/no symptoms). In this study, a high degree of manifestations was estimated to be in 3 of 6 children according to ADOS-2 classification, in 2 children "autism" with a moderate degree of manifestations, in 1 case "autism spectrum" with a moderate degree of manifestations. The group of comparison consisted of 6 children without autism (4 boys and 2 girls) aged from 3 years and 10 months to 7 years. These children did not have any delay in speech development and/or cognitive dysfunction, 4 of them were right-handed, 1 left-handed, 1 ambidexter.

The study was approved by the Ethics Committee of Privolzhsky Research Medical University. Written informed consent was obtained from patients' parents

Magnetic resonance imaging was performed using Magnetom Essenza 1.5 T scanner (Siemens, Germany) provided with 8-channel surface coil for a head. Structural images of the entire brain were obtained with the following parameters: T1 MPR (multiplanar reconstruction) in the sagittal plane; repetition time (TR) $-2160 \mathrm{~ms}$; echo time (TE) $-5.26 \mathrm{~ms}$; flip angle 
(FA) $-15^{\circ}$; field of view (FOV) $-256 \mathrm{~mm}$; matrix $256 \times 256$; voxel size $-1 \times 1 \times 1 \mathrm{~mm}$; scanning time $4 \mathrm{~min} 25 \mathrm{~s}$. The following parameters were used for fMRI: T2* echo-planar sequence; TR - $3000 \mathrm{~ms}$; TE $54 \mathrm{~ms}$; FA - 90'; FOV - $195 \mathrm{~mm}$; matrix - 64×64; slice resolution $-3 \times 3 \mathrm{~mm}$; number of axial slices -25 ; thickness $-4 \mathrm{~mm}$; interslice gap $-1 \mathrm{~mm}$; number of repetitions - 90; time of examination $-4 \min 36 \mathrm{~s}$. Total time of examination was about $25 \mathrm{~min}$.

A block design paradigm was applied during fMRI. It consisted of 9 blocks with $30 \mathrm{~s}$ duration according to the scheme: $\mathrm{B}-\mathrm{A}-\mathrm{B}-\mathrm{A}-\mathrm{B}-\mathrm{A}-\mathrm{B}-\mathrm{A}-\mathrm{B}$ where $\mathrm{B}$ is a background stimulus, $A-$ activation. Tones of different pitch were used as a basic stimulus, and a recorded fairy-tail read by the mother was employed as stimulation. Mother's voice, in this case, was used to interest the child as much as possible and to involve him in the study. Acoustic stimulation was done using MRI compatible earphones.

The data obtained were processed by BrainEx 2.2.0 software (NordicNeuroLab, Norway). The program employed automatically a general linear model, correction of distortion, movements, and eddy currents. Echo-planar images were aligned with structural for more precise localization of the active zones. During the processing, brain activity was extracted in response to the presentation of the speech (fairy-tail) from the activity to the general acoustic stimulus represented by the tones of different pitch which theoretically allows visualization of the areas responsible for speech perception. As a result, individual maps of neuronal activity were obtained for each individual.

For children (three from ASD group and one from the comparison group) unable to stay immovable in the scanner, anesthesiology-aided fMRI was performed. The risk of using anesthesia was assessed to be as class 1-2 according to the American Society of Anesthesiologists physical status classification. All children underwent preanesthetic preparation in the form of a fasting break and placement of a venous catheter. General anesthesia was given in the MRI room using MRI-compatible anesthesia apparatus Dräger Fabius MRI (Dräger, Germany) and a mixture of sevoflurane with oxygen. Introduction of narcosis by sevoflurane inhalation allows medical providers to control the degree of sedation and to present stimuli at the moment of wakening and recovery from narcosis.

Anesthetizing of children was performed in the presence of their parents - this was a mandatory requirement for this procedure. Anesthesia was maintained with sevoflurane at a concentration of $1 \%$ vol., which corresponded to one minimal alveolar concentration, using a supraglottic airway device and a face mask. Respiratory rate and heart rate were monitored during the procedure. Despite the chosen low-flow inhalation, we managed to perform the investigations without artifacts from movement. Five minutes before the beginning of the BOLD sequence, sevoflurane supply was stopped and the patient continued breathing $100 \%$ oxygen via the anesthesia apparatus circuit at a gas flow corresponding to his minute lung ventilation. After $\mathrm{fMRI}$, it took the children 3-5 min to recover from narcosis and 15 min later after the complete recovery they could leave safely the MRI room.

\section{Results}

During speech perception, children with CA as well as children from the comparison group have demonstrated neuronal activation in the superior temporal gyri (STG) with the involvement of the Brodmann areas 22 and/or 41 (acoustical-gnostic sensory speech zones and primary auditory zones). However, the activation pattern in these groups was different. A prominent bilateral activation covering the entire cortical STG area was observed in all children with normal speech development. At the same time, in all CA children lateralization of the perception function was noted (activation occurred mainly in the STG of only one hemisphere whereas, in the second hemisphere, the zones of activation in the STG were minimally prominent and extended or were lacking at all). In 5 children with $\mathrm{CA}$, neuronal activity was lateralized in the right STG and in 1 in the left STG. In this single CA patient with predominantly left neuronal activation (right-hander), the expressive speech consisted of short standard phrases while in the rest 5 children the ability to use a phrase speech was absent. Besides, neuronal activation during speech perception was limited in all children of this group, i.e. did not extend to the entire cortical area of the STG.

Differences of the neuronal activation character during fMRI between the CA children and those without language disorders (uni- or bilateral STG activation, respectively) preserved in performing fMRI under anesthesia. However, in CA children and in children with normal speech development, fMRI under anesthesia showed some reduction of the activated cortical area.

A brief description of four observations of children from the comparison group and children with CA subjected to fMRI examination with and without sedation is given below.

Case 1. Patient K., comparison group, 7 years old, righthander. The child grew and developed in compliance with the age norm. No speech delay was observed; fMRI was performed without sedation. Functional neuroimaging (MRI) data did not show any clinically significant changes. During $f M R I$, active zones in the STG were determined bilaterally (see Figure (a)).

Case 2. Patient F., CA group, 4.5 years old, left-hander. The child was born after the second pregnancy (the first was undeveloped) running with the thread of miscarriage during the entire period and gestosis at 37 weeks' 
gestation. A Cesarian delivery was performed at 38 weeks' gestation. Motor development was without delay. Since his birth he was not very emotional, showed little interest in toys, babbling and prattle were not active. He did not pronounce words, nor did he mimic the adults' actions, he did not use a pointing gesture, made no requests, since his early childhood he preferred the subjects which were not designed for playing (strings, wires), played stereotypically, have no interest in his peers.

The psychic status on examination: attention is attracted but not stable; responds to his name; speech is absent, periodic vocalizations in the form of mooing; understands
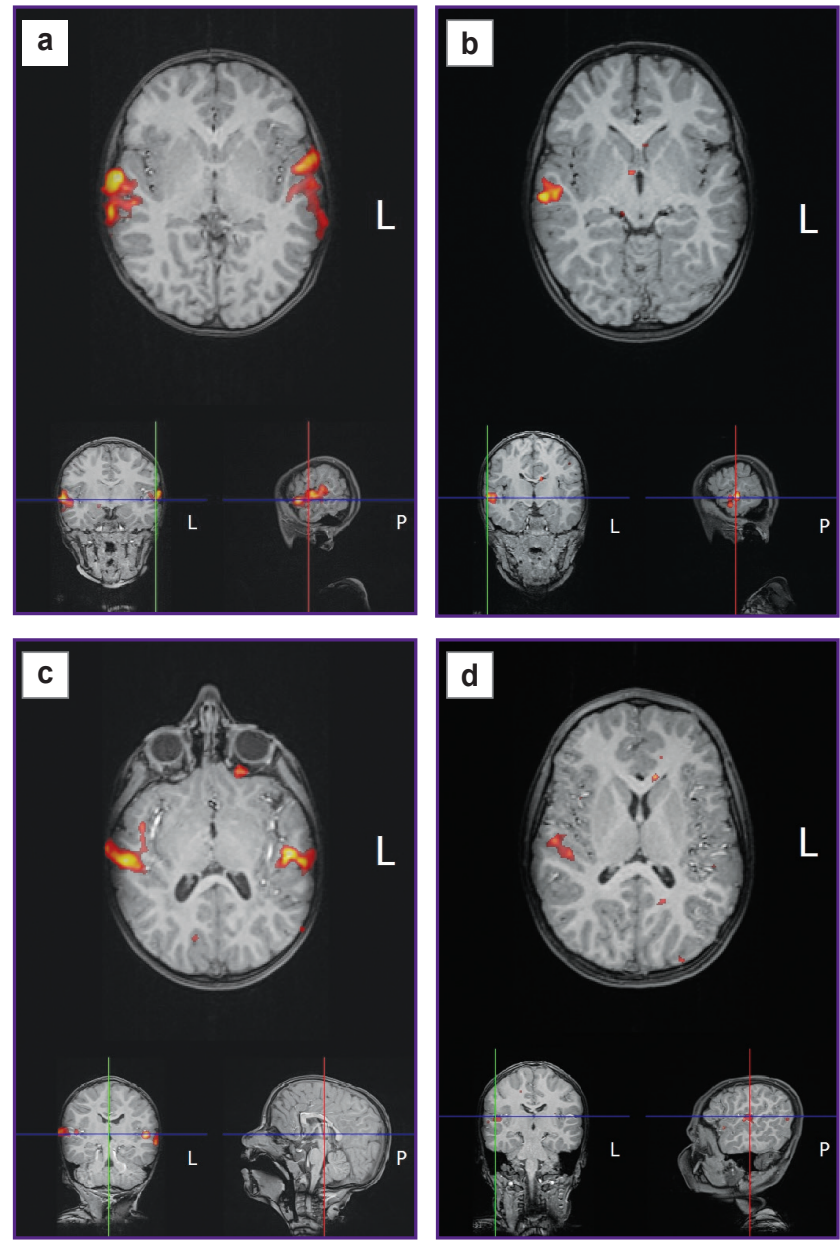

Functional magnetic resonance imaging of the brain, paradigm for speech perception; (a), (b) without sedation; (c), (d) with sevoflurane sedation:

(a) a child without delay of speech development, 7 years old; extended zones of neuronal activity in the projection of the superior temporal gyri are detected bilaterally; (b) a child with autism spectrum disorders, 4.5 years old; an asymmetric limited activation zone is determined in the projection of the right superior temporal gyrus; (c) a child without delay of speech development, 3 years and 10 months; symmetric zones of neuronal activity are seen in the projection of the superior temporal gyri; (d) a child with autism spectrum disorders, 6 years and 8 months; asymmetric zone of activation is determined mainly in the projection of the right superior temporal gyrus simple requests, fulfils them but often several repetitions are required; playing activity is of stereotypic character In the clinical picture, absence of speech and difficulties in social interaction should be referred to the leading symptoms.

Functional MRI investigations were conducted without sedation. Structural MRI did not reveal any significant changes. During fMRI, neuronal activation zone was determined only in the right STG limited by its anterior parts (see Figure (b)).

Case 3. Patient B., comparison group, aged 3 years and 10 months, right-hander. The child with the diagnosis "consequences of hypoxic perinatal trauma of the central nervous system" has been followed-up by a neurologist since 1 month of age. On examination: there is a delay in the motor development which served as a ground for administering $\mathrm{fMRI}$ brain examination. However, no delay in speech development and cognitive activity are observed in the child, no signs of autism are present either.

Functional MRI examination was conducted with sedation. Structural MRI revealed the picture of periventricular gliotic changes probably of post-hypoxic character and hypogenesis of the corpus callosum. During fMRI, bilateral activation zones were detected in STG though the limitation of activation areas was noted being conceivably the effect of anesthesia (see Figure (c)).

Case 4. Patient M., group of CA patients, 6 years and 8 months, right-hander. The child was born after the third pregnancy (the first pregnancy ended with spontaneous abortion, the second with intrauterine fetus death). Delivery occurred at 35 weeks' gestation, a long period after water breaking was fixed. At birth the baby's mass was $2800 \mathrm{~g}$, length $48 \mathrm{~cm}$, Apgar score 7/8.

Motor development goes without delay. Neuropsychic development: the child was emotional, interested in toys, though babbling and baby's prattle were weak, by the age of 1 year speech did not yet appear, a pointing gesture was not formed. From 1.5 to 2 years, specific features of his behavior were gradually increasing: he stopped responding to the name, movement stereotypes in the form of waving hands and circus movement appeared. He did not understand the speech addressed to him, did not fulfil instructions. His peers did not interest him. He started to pronounce his first words at the age of 5 years. Hyperactivity and movement stereotypes persisted.

On examination: a small number of separate words, which he does not practically use spontaneously, can make a request (for example, "I want to drink") when his mother asks him to do, does not use words himself when asking for something. Sound pronunciation and speech rhythm are impaired, the major part of the pronounced words are echolalias. He is constantly vocalizing (emitting strange monotonous sounds, which are not words or something similar to words), does not use these vocalizations for communication.

Structural MRI did not reveal any clinically significant 
changes. During fMRI, the activation zone was determined only in the region of the area-limited right STG (see Figure (d)).

\section{Discussion}

The FMRI examination performed by us showed that changes in neuronal response to the speech perception were observed in the preschool children with $\mathrm{CA}$ in comparison with the children free from speech disorders. These changes in autistic children manifested themselves in a single-sided (lateralized) and limited involvement of the auditory cortex though the correlation between the side of activation and the dominant hand has not been detected. At the same time, in all children from the comparison group, a symmetric activation in both STGs extended along the entire length of the gyrus was obtained during presentation of the paradigm for speech perception.

The phenomenon of function lateralization has been studied especially actively in recent years. A different role of the right and left STG in the auditory gnosis has been demonstrated in the study of 20 healthy preschool children [39]. Having performed fMRI with the paradigms for voice recognition and recognition of similarly sounding tones the authors showed that the right STG is responsible for extraction of acoustic signs enabling the recognition of the voice. In $\mathrm{ASD}$, the phenomenon of cerebral lateralization has been considered from different positions. So, in people with the diseases of this group, a more frequent occurrence of left-handers and ambidexters has been revealed [40]. Another group of scientists demonstrated the interhemispheric organization disorder seen in lateralization of the muscle tone and spontaneous gesticulation in children with ASD [41]. Investigating speech perception by 2-3-year children Redcay [34] showed the predominant involvement of the right hemisphere structures in ASD patients. They compared and analyzed normally played and inversely reproduced speech within the frames of a single paradigm. Excitation of the temporal gyrus predominantly in one of the hemispheres during speech perception in patients with these diseases demonstrated in our work can be explained by some disorders of connectivity including interhemispheric as well. Besides, taking into account predominantly rightsided activation, we can suppose that a child with CA responds mainly to his own voice rather than to the speech since, as the fundamental investigations show, it is the left STG that is responsible for the recognition of the word meaning [39].

Our study has also verified the possibility of using sevoflurane inhalation narcosis for successful fRMI performance with passive paradigms. Sedation with sevoflurane during $\mathrm{fMRI}$ in both groups did not lead to changes in the lateralization pattern of neuronal activation.

\section{Conclusion}

Application of recent specialized method of neurovisualization such as magnetic resonance imaging allows neurologists to explore those features of brain functioning that are not detected by standard methods in patients with delayed psychic development. Our observations have also demonstrated the possibility of using functional magnetic resonance imaging with the paradigm for speech understanding under general anesthesia in order to detect individual features of brain functioning in children with autistic spectrum disorders. The drawback of our study is a small number of observations and clinical variants of autism. Besides, the current investigation should be supplemented with morphometric data and mapping information which can prove the interconnection disorder between the speech centers and the asymmetry in the development of different structures. It is also necessary to continue observations of patients which would enable the establishment of clinical and neurovisualization parallels and the possibility of using findings of functional magnetic resonance imaging as prognostic biomarkers.

Study funding and conflicts of interest. The work was not supported by any financial sources and there are no conflicts of interest related to this study.

\section{References}

1. Simashkova N.V., Makushkin E.V. Rasstroystva autisticheskogo spektra: diagnostika, lechenie, nablyudenie. Klinicheskie rekomendatsii (protokol lecheniya) [Autism spectrum disorders: diagnosis, treatment, monitoring. Clinical guidelines (treatment protocol)]. 2015.

2. American Psychiatric Association. Diagnostic and statistical manual of mental disorders. American Psychiatric Association; 2013, https://doi.org/10.1176/appi. books. 9780890425596.

3. Ecker C., Spooren W., Murphy D.G.M. Translational approaches to the biology of autism: false dawn or a new era? Mol Psychiatry 2012; 18(4): 435-442, https://doi.org/10.1038/ mp.2012.102.

4. Haigh S.M., Heeger D.J., Dinstein I., Minshew N., Behrmann M. Cortical variability in the sensory-evoked response in autism. J Autism Dev Disord 2014; 45(5): 11761190, https://doi.org/10.1007/s10803-014-2276-6.

5. Geschwind D.H., Levitt P. Autism spectrum disorders: developmental disconnection syndromes. Curr Opin Neurobiol 2007; 17(1): 103-111, https://doi.org/10.1016/j. conb.2007.01.009.

6. Ha S., Sohn I.-J., Kim N., Sim H.J., Cheon K.-A. Characteristics of brains in autism spectrum disorder: structure, function and connectivity across the lifespan. Exp Neurobiol 2015; 24(4): 273, https://doi.org/10.5607/en.2015.24.4.273.

7. Bernhardt B.C., Di Martino A., Valk S.L., Wallace G.L. Neuroimaging-based phenotyping of the autism spectrum. Curr Top Behav Neurosci 2017; 30: 341-355, https://doi. org/10.1007/7854_2016_438. 
8. Berg A.T., Dobyns W.B. Progress in autism and related disorders of brain development. Lancet Neurol 2015; 14(11): 1069-1070, https://doi.org/10.1016/s1474-4422(15) 00048-4.

9. Ismail M.M., Keynton R.S., Mostapha M.M., ElTanboly A.H., Casanova M.F., Gimel'farb G.L., El-Baz A. Studying autism spectrum disorder with structural and diffusion magnetic resonance imaging: a survey. Front Hum Neurosci 2016; 10: 211, https://doi.org/10.3389/fnhum.2016.00211.

10. Heeger D.J., Ress D. What does fMRI tell us about neuronal activity? Nat Rev Neurosci 2002; 3(2): 142-151, https://doi.org/10.1038/nrn730.

11. Logothetis N.K., Wandell B.A. Interpreting the BOLD signal. Annu Rev Physiol 2004; 66(1): 735-769, https://doi org/10.1146/annurev.physiol.66.082602.092845.

12. Logothetis N.K. The neural basis of the blood-oxygenlevel-dependent functional magnetic resonance imaging signal. Philos Trans R Soc Lond B Biol Sci 2002; 357(1424): 1003-1037, https://doi.org/10.1098/rstb.2002.1114.

13. Seliverstova E.V., Seliverstov Yu.A., Konovalov R.N., Illarioshkin S.N. Resting-state fMRI: new possibilities for studying physiology and pathology of the brain. Annaly kliniceskoj i eksperimental'noj nevrologii 2013; 7(4): 39-44.

14. Seliverstov Y.A., Seliverstova E.V., Konovalov R.N., Klyushnikov S.A., Krotenkova M.V., Illarioshkin S.N. Clinical and imaging analysis of huntington disease with use of restingstate functional magnetic resonance imaging. The Neurological Journal 2015; 20(3): 11, https://doi.org/10.18821/1560-95452015-20-3-11-21.

15. Dichter G.S. Functional magnetic resonance imaging of autism spectrum disorders. Dialogues Clin Neurosci 2012; 14(3): 319-351.

16. Failla M.D., Moana-Filho E.J., Essick G.K., Baranek G.T., Rogers B.P., Cascio C.J. Initially intact neural responses to pain in autism are diminished during sustained pain. Autism 2017; 22(6): 669-683, https://doi. org/10.1177/1362361317696043.

17. Gu X., Zhou T.J., Anagnostou E., Soorya L., Kolevzon A., Hof P.R., Fan J. Heightened brain response to pain anticipation in high-functioning adults with autism spectrum disorder. Eur J Neurosci 2017; 47(6): 592-601, https://doi.org/10.1111/ejn.13598.

18. Carlisi C.O., Norman L., Murphy C.M., Christakou A., Chantiluke K., Giampietro V., Simmons A., Brammer M. Murphy D.G., Mataix-Cols D., Rubia K.; MRC AIMS consortium. Shared and disorder-specific neurocomputational mechanisms of decision-making in autism spectrum disorder and obsessivecompulsive disorder. Cereb Cortex 2017; 27(12): 5804-5816, https://doi.org/10.1093/cercor/bhx265

19. Stanfield A.C., Philip R.C.M., Whalley H., Romaniuk L., Hall J., Johnstone E.C., Lawrie S.M. Dissociation of brain activation in autism and schizotypal personality disorder during social judgments. Schizophr Bull 2017; 43(6): 1220-1228, https://doi.org/10.1093/schbul/sbx083.

20. Murphy C.M., Christakou A., Giampietro V., Brammer M., Daly E.M., Ecker C., Johnston P., Spain D., Robertson D.M.; MRC AIMS Consortium, Murphy D.G., Rubia K. Abnormal functional activation and maturation of ventromedial prefrontal cortex and cerebellum during temporal discounting in autism spectrum disorder. Hum Brain Mapp 2017; 38(11): 5343-5355, https://doi.org/10.1002/ hbm.23718.

21. Philip R.C., Dauvermann M.R., Whalley H.C.,
Baynham K., Lawrie S.M., Stanfield A.C. A systematic review and meta-analysis of the $\mathrm{fMRI}$ investigation of autism spectrum disorders. Neurosci Biobehav Rev 2012; 36(2): 901-942, https://doi.org/10.1016/j.neubiorev.2011.10.008.

22. Kim S.Y., Choi U.S., Park S.Y., Oh S.H., Yoon H.W., Koh Y.J., Im W.Y., Park J.I., Song D.H., Cheon K.A., Lee C.U. Abnormal activation of the social brain network in children with autism spectrum disorder: an fMRI study. Psychiatry Investig 2015; 12(1): 37, https://doi.org/10.4306/pi.2015.12.1.37.

23. Di Martino A., Ross K., Uddin L.Q., Sklar A.B., Castellanos F.X., Milham M.P. Functional brain correlates of social and nonsocial processes in autism spectrum disorders: an activation likelihood estimation meta-analysis. Biol Psychiatry 2009; 65(1): 63-74, https://doi.org/10.1016/j. biopsych.2008.09.022.

24. Lombardo M.V., Pierce K., Eyler L.T., Carter Barnes C., Ahrens-Barbeau C., Solso S., Campbell K., Courchesne E. Different functional neural substrates for good and poor language outcome in autism. Neuron 2015; 86(2): 567-577, https://doi.org/10.1016/j.neuron.2015.03.023.

25. Wang A.T., Lee S.S., Sigman M., Dapretto M. Neural basis of irony comprehension in children with autism: the role of prosody and context. Brain 2006; 129(4): 932-943, https:// doi.org/10.1093/brain/awl032.

26. Redcay E., Courchesne E. Deviant functional magnetic resonance imaging patterns of brain activity to speech in 2-3-year-old children with autism spectrum disorder. Biol Psychiatry 2008; 64(7): 589-598, https://doi.org/10.1016/j. biopsych.2008.05.020.

27. Kana R.K., Keller T.A., Cherkassky V.L., Minshew N.J., Just M.A. Sentence comprehension in autism: thinking in pictures with decreased functional connectivity. Brain 2006; 129(9): 2484-2493, https://doi.org/10.1093/ brain/awl164.

28. Lee Y., Park B., James O., Kim S.-G., Park H. Autism spectrum disorder related functional connectivity changes in the language network in children, adolescents and adults. Front Hum Neurosci 2017; 11: 418, https://doi.org/10.3389/ fnhum.2017.00418.

29. Chouinard B., Volden J., Cribben I., Cummine J. Neurological evaluation of the selection stage of metaphor comprehension in individuals with and without autism spectrum disorder. Neuroscience 2017; 361: 19-33, https://doi. org/10.1016/j.neuroscience.2017.08.001.

30. Knaus T.A., Burns C., Kamps J., Foundas A.L. Atypical activation of action-semantic network in adolescents with autism spectrum disorder. Brain Cogn 2017; 117: 57-64, https://doi.org/10.1016/j.bandc.2017.06.004.

31. Yang J., Lee J. Different aberrant mentalizing networks in males and females with autism spectrum disorders: evidence from resting-state functional magnetic resonance imaging. Autism 2016; 22(2): 134-148, https://doi. org/10.1177/1362361316667056.

32. Lenroot R.K., Yeung P.K. Heterogeneity within autism spectrum disorders: what have we learned from neuroimaging studies? Front Hum Neurosci 2013; 7, https://doi.org/10.3389/ fnhum.2013.00733.

33. Andrews D.S., Marquand A., Ecker C., McAlonan G. Using pattern classification to identify brain imaging markers in autism spectrum disorder. Curr Top Behav Neurosci 2018; 40: 413-436, https://doi.org/10.1007/7854_2018_47.

34. Redcay E. The superior temporal sulcus performs a common function for social and speech perception: 
implications for the emergence of autism. Neurosci Biobehav Rev 2008; 32(1): 123-142, https://doi.org/10.1016/j.neubiorev. 2007.06.004.

35. Pierce K. Early functional brain development in autism and the promise of sleep fMRI. Brain Res 2011; 1380: 162174, https://doi.org/10.1016/j.brainres.2010.09.028.

36. Tang C.Y., Ramani R. fMRI and anesthesia. Int Anesthesiol Clin 2016; 54(1): 129-142, https://doi.org/10.1097/ aia.0000000000000081.

37. Gemma M., Scola E., Baldoli C., Mucchetti M., Pontesilli S., De Vitis A., Falini A., Beretta L. Auditory functional magnetic resonance in awake (nonsedated) and propofolsedated children. Paediatr Anaesth 2016; 26(5): 521-530, https://doi.org/10.1111/pan.12884.

38. Gemma M., Scola E., Baldoli C., Mucchetti M., Pontesilli S., De Vitis A., Falini A., Beretta L. Brain restingstate functional connectivity is preserved under sevoflurane anesthesia in patients with pervasive developmental disorders: a pilot study. Brain Connect 2017; 7(4): 250-257, https://doi. org/10.1089/brain.2016.0448.

39. Raschle N.M., Smith S.A., Zuk J., Dauvermann M.R., Figuccio M.J., Gaab N. Investigating the neural correlates of voice versus speech-sound directed information in preschool children. PLoS One 2014; 9(12): e115549, https://doi. org/10.1371/journal.pone.0115549.

40. Markou P., Ahtam B., Papadatou-Pastou M. Elevated levels of atypical handedness in autism: meta-analyses. Neuropsychol Rev 2017; 27(3): 258-283, https://doi. org/10.1007/s11065-017-9354-4.

41. Paquet A., Golse B., Girard M., Olliac B., Vaivre-Douret L. Laterality and lateralization in autism spectrum disorder, using a standardized neuro-psychomotor assessment. Dev Neuropsychol 2017; 42(1): 39-54, https://doi.org/10.1080/ 87565641.2016.1274317. 